\title{
Rechallenge of ponatinib in chronic myeloid leukaemia after hepatotoxicity
}

YL Boo ${ }^{1 *}$, MD, MRCP (UK), Christopher CK Liam², MD, MRCP (UK), SG Toh¹, MRCP (UK), SM Lim¹, MRCP (UK)

${ }^{1}$ Department of Haematology, Hospital Sultanah Aminah, Johor Bahru, Malaysia

${ }^{2}$ Department of Haematology, Hospital Ampang, Selangor, Malaysia

Hong Kong Med J 2019;25:162-3

*Corresponding author: coolrontin@gmail.com

https://doi.org/10.12809/hkmj187420

\section{Commentary}

Chronic myeloid leukaemia (CML) is a myeloproliferative neoplasm associated with an oncogenic fusion gene breakpoint cluster regionAbelson (BCR-ABL) encoding a protein with tyrosine kinase activity. Tyrosine kinase inhibitors (TKIs) have revolutionised the treatment and improved overall survival in patients with CML. Nonetheless, first- and second-generation TKIs are ineffective against BCR-ABL T315I mutation. This has led to the development of a third-generation inhibitor, ponatinib. Ponatinib can cause hepatotoxicity including fulminant hepatic failure and even deaths. ${ }^{1}$ We recently encountered a case of successful rechallenge with ponatinib following severe ponatinib-induced hepatotoxicity that resolved with corticosteroid. A 30-year-old man diagnosed with CML was started on ponatinib $45 \mathrm{mg}$ once daily after the discovery of BCR-ABL T315I mutation. Despite initially responding well, the patient developed hepatotoxicity after 6 months of treatment. The patient's alanine aminotransferase level was 5 times the upper normal limit, and his total bilirubin and alkaline phosphatase levels were normal. There were no other significant identifiable causes for the underlying liver impairment. The ponatinib dose was reduced, but the patient's alanine aminotransferase level increased further, and he developed conjugated hyperbilirubinaemia within 2 weeks. Ponatinib was subsequently withheld, but the patient developed intense pruritus and deep jaundice. Ultrasound scan of the hepatobiliary system and endoscopic ultrasonography was normal, autoimmune and viral hepatitis screening tests were negative, and liver biopsy was consistent with mild steatohepatitis. Oral prednisolone was started at $40 \mathrm{mg}$ daily, and the patient's liver function showed marked improvement after 2 weeks of treatment and normalised within 1 month of tapering steroids (Fig). The patient was rechallenged with ponatinib at a lower dose; this was tolerated well and liver function was normal during subsequent review. Ponatinib was gradually escalated during 6 months of follow-up without any adverse events. Ponatinib, a third-generation TKI, has been

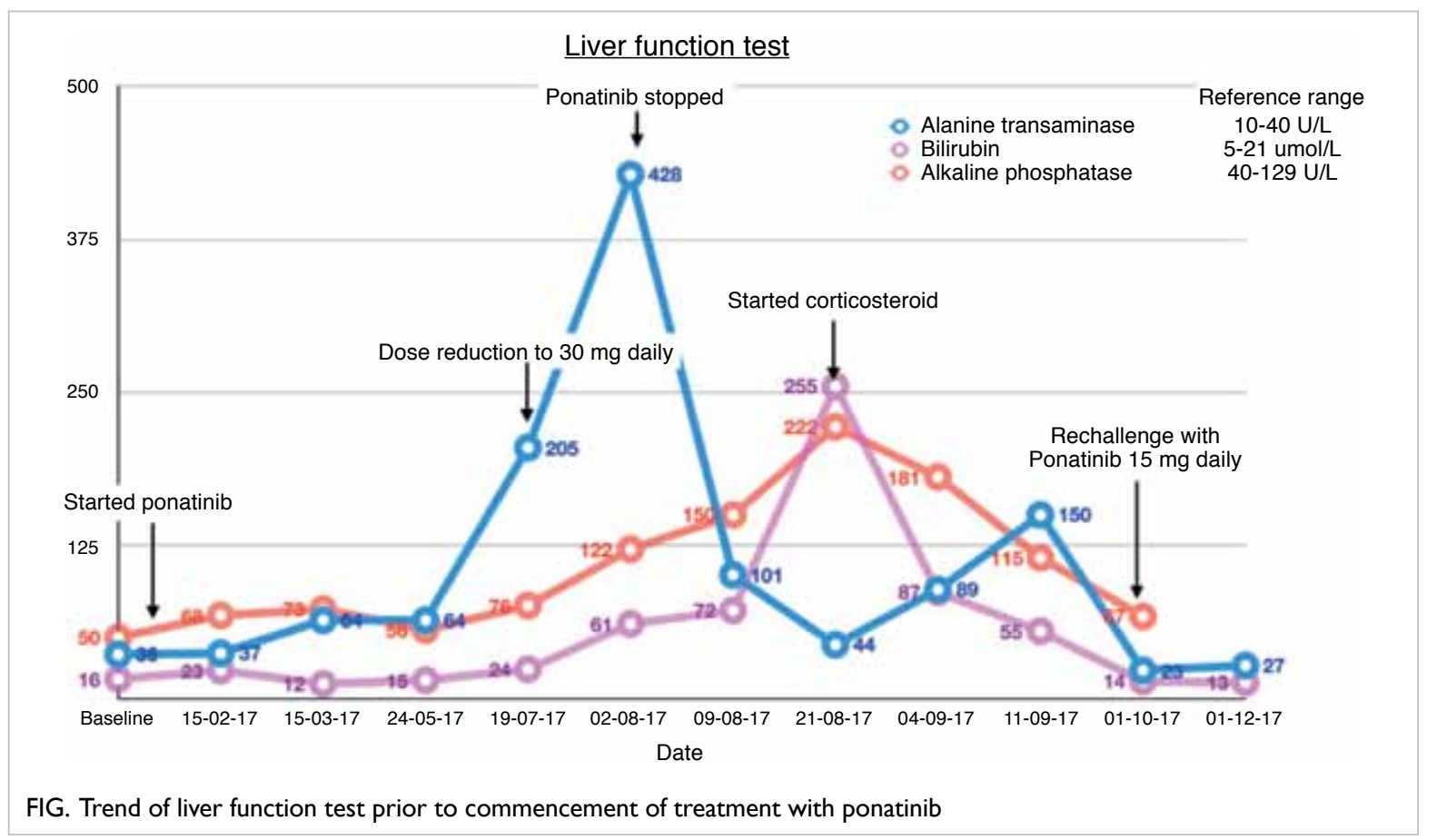


shown to be effective against T315I mutation. A phase II clinical trial evaluated ponatinib in patients resistant or intolerant to nilotinib or dasatinib, or who had T315I mutation produced a major cytogenetic response in $60 \%$ of patients with chronicphase CML. It also achieved major haematological response in $52 \%$ of patients with accelerated-phase CML, 31\% of patients with blast-phase CML, and $41 \%$ of Philadelphia chromosome-positive patients with acute lymphoblastic leukaemia. ${ }^{2}$ However, the therapeutic efficacy of ponatinib needs to be weighed carefully against the risks before commencement of treatment. Cardiovascular safety profile, hepatotoxicity, pancreatitis, and cytopenias are among the serious adverse events that may lead to significant morbidity and mortality. ${ }^{3}$

The incidence of serum alanine aminotransferase and aspartate aminotransferase elevations has been reported as $56 \%$ for all grades, and $8 \%$ for Grades 3 or $4 .{ }^{1}$ These adverse events were graded based on National Cancer Institute Common Terminology Criteria for Adverse Events, version 4.0. ${ }^{4}$ The presence of serum aminotransferase levels above 3 times the normal upper limit should lead to dose reduction or temporary cessation of treatment, with resumption at a lower dose once levels normalise. In patients with clinically apparent liver injury and jaundice, treatment should be discontinued. ${ }^{1}$ In a phase I trial of ponatinib, observed adverse events, which included hepatotoxicity, were dosedependent and reported as self-limiting in most of the events. ${ }^{4}$ Subsequent rechallenge of ponatinib at lower dose was generally successful; however, Grade 4 adverse events in some patients warranted cessation of treatment for these patients. ${ }^{4}$ Successful reversion of imatinib-induced hepatotoxicity in CML patients has been previously reported. ${ }^{5} \mathrm{We}$ observed rapid improvement in our patient's liver function following corticosteroid therapy, and thus managed to avoid permanent discontinuation of ponatinib in the treatment of T315I mutation CML. The commencement of ponatinib at lower dose is recommended, with gradual escalation of dosage as tolerated by the patient. In preclinical studies, at doses of $>30 \mathrm{mg}$, trough blood concentrations were reported to completely suppress the emergence of BCR-ABL mutations. ${ }^{4}$

The pathogenic mechanisms of ponatinibinduced hepatotoxicity are not fully understood. However, the histopathology findings of steatohepatitis and its response to corticosteroid suggested an inflammatory response; this has been previously described in imatinib-induced hepatotoxicity. ${ }^{6}$ The clinical finding of severe hepatic impairment was disproportionate to the liver biopsy findings in our patient, possibly partly contributed to by the commencement of corticosteroid therapy before biopsy was carried out. As the patient had no significant past medical illness to suggest nonalcoholic steatohepatitis, we were unable to conclude its relationship with the development of hepatic impairment in our patient.

Corticosteroid therapy for severe hepatotoxicity and steatohepatitis induced by ponatinib has not, to our knowledge, been previously reported. Corticosteroid therapy and rechallenge of ponatinib have potential for successfully treating patients with severe ponatinib-induced hepatotoxicity and T315I mutation CML.

\section{Author contributions}

All authors had full access to the data, contributed to the study, approved the final version for publication, and take responsibility for its accuracy and integrity.

Concept or design: All authors.

Acquisition of data: YL Boo, SG Toh.

Analysis or interpretation of data: YL Boo, CCK Liam.

Drafting of the manuscript: YL Boo, CCK Liam.

Critical revision for important intellectual content: All authors.

\section{Acknowledgement}

The authors would like to thank the Director General of Health for permission to publish this article.

\section{Conflicts of interest}

All authors have disclosed no conflicts of interest.

\section{Funding/support}

This research received no specific grant from any funding agency in the public, commercial, or not-for-profit sectors.

\section{Patient consent}

This study was conducted in accordance with the Declaration of Helsinki. The patient provided written informed consent for publication.

\section{References}

1. Iclusig [package insert]. Cambridge, MA: Ariad Pharmaceuticals; 2012.

2. Kantarjian HM, Pinilla-Ibarz J, Le Coutre PD, et al. Five-year results of the ponatinib phase II PACE trial in heavily pretreated CP-CML patients (pts). J Clin Oncol 2017;35(Suppl 15):7012.

3. Price KE, Saleem N, Lee G, Steinberg M. Potential of ponatinib to treat chronic myeloid leukemia and acute lymphoblastic leukemia. Onco Targets Ther 2013;6:1111-8.

4. Cortes JE, Kantarjian H, Shah NP, et al. Ponatinib in refractory Philadelphia chromosome-positive leukemias. N Engl J Med 2012;367:2075-88.

5. Ferrero D, Pogliani EM, Rege-Cambrin G, et al. Complete reversion of imatinib-induced hepatotoxicity in chronic myeloid leukemia patients by low-intermediate dose corticosteroid. Blood 2005;106:4856.

6. Ferrero D, Pogliani EM, Rege-Cambrin G, et al. Corticosteroids can reverse severe imatinib-induced hepatotoxicity. Haematologica 2006;91(6 Suppl):ECR27. 